International Mathematical Forum, 1, 2006, no. 13, 623-627

\title{
ON HELICES IN THE DOUBLY ISOTROPIC SPACE $I_{3}^{(2)}$
}

\author{
A. O. Öğrenmiş, M. Bektaş, M. Ergüt \\ Department of Mathematics \\ Firat University \\ 23119 Elazı̆̆ , Turkey
}

\begin{abstract}
T. Ikawa obtained in [4] the following characteristic ordinary differential equation

$$
\nabla_{X} \nabla_{X} \nabla_{X} X-K \nabla_{X} X=0, \quad K=k^{2}-\tau^{2}
$$

for the circular helix which corresponds to the case that the curvatures $k$ and $\tau$ of a time-like curve $c$ on the Lorentzian manifold $\mathrm{M}$ are constant.

N. Ekmekçi and H. H. Hacisalihoglu generalized in [3] T. Ikawa's this result, i.e. $k(s)$ and $\tau(s)$ are variable, but $\frac{k(s)}{\tau(s)}$ is constant.

In [1] H.Balgetir, M.Bektas and M.Ergüt obtained a geometric characterization of Null Frenet curve with constant ratio of curvature and torsion (called null general helix).

In this paper, making use of method in $[1,3,4]$, we obtained characterizations of a curve with respect to the Frenet frame of Doubly Isotropic Space $I_{3}^{(2)}$.
\end{abstract}

\section{Mathematics Subject Classification: 53B30}

Keywords: Doubly Isotropic Space, General Helix

\section{Introduction}

Differential gometry of Doubly Isotropic Space $I_{3}^{(2)}$ has been studied in detail in [2],[5] and [6].

The absolute of this space consists of a plane $w$ (the absolute plane), a line $f \subset w$ (the absolute line) and a point $U \in f$ (the absolute point). Projective transformations preserving the absolute $(w, f, U)$ form a group which is called the similarity group in $I_{3}^{(2)}$. This group can be written in nonhomogeneous coordinates in the form 


$$
\begin{gathered}
x^{\prime}=a_{1} x+a_{2}, \\
y^{\prime}=b_{1} x+b_{2} y+b_{3}, \\
z^{\prime}=c_{1} x+c_{2} y+c_{3} z+c_{4}
\end{gathered}
$$

where $a_{i}, b_{j}, c_{k}(i=1,2 ; j=1,2,3 ; k=1,2,3,4)$ are real constants.

The subgroup of this group defined by $a_{1}=b_{2}=c_{3}=1$ is the isometry group of space $I_{3}^{(2)}$.

In this paper we shall use notations from $[2,6]$.

Let now $c: I \rightarrow I_{3}^{(2)}, I \subseteq R$ be a curve given in coordinates by

$$
x=s, y=y(s), z=z(s),
$$

where $s$ is the invariant parameter which in the geometry of the space $I_{3}^{(2)}$ coincides with the abscisa of the curve's point. Derivatives with respect to $s$ will be denoted by primes.

To any curve of the class $C^{r}(r \geq 3)$ the Frenet trihedron $\{T(s), N(s), B(s)\}$ is assigned where

$$
T(s)=\left(1, y^{\prime}(s), z^{\prime}(s)\right), N(s)=\left(0,1, \frac{z^{\prime \prime}(s)}{y^{\prime \prime}(s)}\right), B(s)=(0,0,1)
$$

$T(s)$ is called the unit tangent vector, $N(s)$ the unit principal normal vector and $B(s)$ the unit binormal vector.

The curvature $k(s)$ and the torsion $\tau(s)$ of the curve $c$ are defined by

$$
k(s)=y^{\prime \prime}(s), \quad \tau(s)=\left(\frac{z^{\prime \prime}(s)}{y^{\prime \prime}(s)}\right)^{\prime} .
$$

For these vector fields the following Frenet formulas hold

$$
\begin{gathered}
\nabla_{T_{(s)}} T(s)=k(s) N(s), \\
\nabla_{T_{(s)}} N(s)=\tau(s) B(s), \\
\nabla_{T_{(s)}} B(s)=0 .
\end{gathered}
$$

\section{The Characterizations of Curves on Doubly Isotropic Space.}

Definition 2.1. Let $c$ be a curve of Doubly Isotropic Space $I_{3}^{(2)}$ and $\{T(s), N(s), B(s)\}$ be the Frenet frame of Doubly Isotropic Space $I_{3}^{(2)}$ along $c$. If $k$ and $\tau$ are positive constants along $c$, then $c$ is called a circular helix with respect to the Frenet frame. 
Definition 2.2. Let $c$ be a curve of Doubly Isotropic Space $I_{3}^{(2)}$ and $\{T(s), N(s), B(s)\}$ be the Frenet frame of Doubly Isotropic Space $I_{3}^{(2)}$ along $c$. A curve $c$ such that

$$
\frac{k(s)}{\tau(s)}=\text { const }
$$

is called a general helix with respect to Frenet frame.

Theorem 2.1. Let $c$ be a curve of Doubly Isotropic Space $I_{3}^{(2)}$. $c$ is a general helix with respect to the Frenet frame $\{T(s), N(s), B(s)\}$ if and only if

$$
\nabla_{T_{(s)}} \nabla_{T_{(s)}} \nabla_{T_{(s)}} T(s)-K(s) \nabla_{T_{(s)}} T(s)=3 k^{\prime}(s) \nabla_{T_{(s)}} N(s)
$$

where $K(s)=\frac{k^{\prime \prime}(s)}{k(s)}$.

Proof. Suppose that $c$ is general helix with respect to the Frenet frame $\{T(s), N(s), B(s)\}$. Then from (1.3), we have

$$
\nabla_{T_{(s)}} \nabla_{T_{(s)}} \nabla_{T_{(s)}} T(s)=k^{\prime \prime}(s) N(s)+\left(2 k^{\prime}(s) \tau(s)+k(s) \tau^{\prime}(s)\right) B(s) .
$$

Now, since $c$ is general helix with respect to the Frenet frame

$$
\frac{k(s)}{\tau(s)}=\text { const }
$$

and this upon the derivation gives rise to

$$
k^{\prime}(s) \tau(s)=k(s) \tau^{\prime}(s)
$$

If we substitute the equations (2.3),

$$
N(s)=\frac{1}{k(s)} \nabla_{T_{(s)}} T(s)
$$

and

$$
B(s)=\frac{1}{\tau(s)} \nabla_{T_{(s)}} N(s)
$$

in (2.2), we obtain (2.1).

Conversely let us assume that the equation (2.1) holds. We show that the curve $c$ is a general helix. Differentiating covariantly (2.4) we obtain

$$
\nabla_{T_{(s)}} N(s)=-\frac{k^{\prime}(s)}{k^{2}(s)} \nabla_{T_{(s)}} T(s)+\frac{1}{k(s)} \nabla_{T_{(s)}} \nabla_{T_{(s)}} T(s)
$$

and so

$$
\nabla_{T_{(s)}} \nabla_{T_{(s)}} N(s)=\left(-\frac{k^{\prime}(s)}{k^{2}(s)}\right)^{\prime} \nabla_{T_{(s)}} T(s)-2 \frac{k^{\prime}(s)}{k^{2}(s)} \nabla_{T_{(s)}} \nabla_{T_{(s)}} T(s)
$$




$$
+\frac{1}{k(s)} \nabla_{T_{(s)}} \nabla_{T_{(s)}} \nabla_{T_{(s)}} T(s) .
$$

If we use (2.1) in (2.7) and make some calculations, we have

$$
\begin{gathered}
\nabla_{T_{(s)}} \nabla_{T_{(s)}} N(s)=\left[\left(-\frac{k^{\prime}(s)}{k^{2}(s)}\right)^{\prime}+\frac{K(s)}{k(s)}\right] \nabla_{T_{(s)}} T(s)-2 \frac{\left(k^{\prime}(s)\right)^{2}}{k^{2}(s)} N(s) \\
+\frac{k^{\prime}(s) \tau(s)}{k(s)} B(s) .
\end{gathered}
$$

Also we obtain

$$
\nabla_{T_{(s)}} \nabla_{T_{(s)}} N(s)=\tau^{\prime}(s) B(s)
$$

since (2.8) and (2.9) are equal, routine calculations show that $c$ is a general helix.

Theorem 2.2. Let $c$ be a curve of Doubly Isotropic Space $I_{3}^{(2)}$. $c$ is a general helix with respect to the Frenet frame $\{T(s), N(s), B(s)\}$, if and only if

$$
\nabla_{T_{(s)}} \nabla_{T_{(s)}} \nabla_{T_{(s)}} T(s)-K(s) \nabla_{T_{(s)}} T(s)=3 \lambda \tau^{\prime}(s) \nabla_{T_{(s)}} N(s)
$$

where $K(s)=\frac{k^{\prime \prime}(s)}{k(s)}$ and $\lambda=\frac{k(s)}{\tau(s)}=$ const.

Proof. It is similar to the proof of Theorem 2.1.

Corollary 2.1. Let $c$ be a curve of Doubly Isotropic Space $I_{3}^{(2)}$. $c$ is a circular helix with respect to the Frenet frame $\{T(s), N(s), B(s)\}$, if and only if

$$
T(s)=\frac{1}{2} s^{2}+s+d
$$

where $d$ is a constant

Proof. From the hypotesis of corollary 2.1 and since $c$ is a circular helix, we can show easily (2.11).

\section{References}

[1] Balgetir, H., Bektas, M., and Ergüt, M., On a Characterization of Null Helix, Bull. Ins. Math. Aca. Sin.29 (1) (2001), 71-78.

[2] Brauner, H., Geometrie Des Zweifach Isotropen Raumes. II, J. Reine U. Angew.Math.266 (1967), 132-158.

[3] Ekmekçi, N. and Hacisalihoglu, H.H., On Helices of a Lorentzian manifold, Commun. Fac. Sci., Üniv. Ank. Series A1 (1996), 45-50.

[4] Ikawa, T, On Curves and Submanifolds in an Indefinite-Riemannian Manifold, Tsukuba J. Math., 9 (1985), 353-371.

[5] Kallenberg, G., Differential Geometry of a Particular Group of Projective Transformations, Proc. Nederl. Akad. Wetens. Proc. A 60 (1957), 147-158. 
[6] Pavkoviç, B. and Kamenaroviç, I., The General Solution of The Frenet System In The Doubly Isotropic Space $I_{3}^{(2)}$, Rad JAZU knj.428, Mat. Znan. 6 (1987), 17-24.

Received: September 14, 2005 